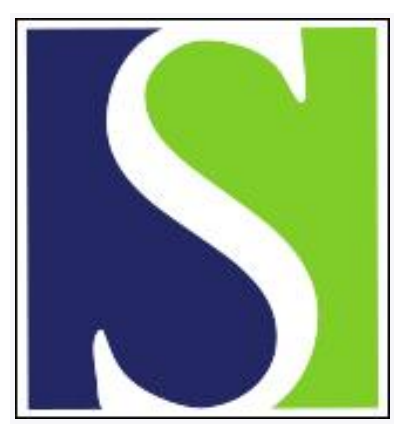

Scand J Work Environ Health 2000;26(5):390-397

https://doi.org/10.5271/sjweh.559

Issue date: Oct 2000

Differences between work methods and gender in computer mouse use

by Wahlström J, Svensson J, Hagberg M, Johnson PW

The following articles refer to this text: 2003;29(3):197-205; SJWEH Supplements 2007;(3):26-32; SJWEH Supplements 2007;(3):33-41; 2009;35(2):113-126; 2009;35(2):85-95

Key terms: electrogoniometer; electromyography; force; gender difference; input device; subjective rating; video display terminal; video display unit

This article in PubMed: www.ncbi.nlm.nih.gov/pubmed/11103837

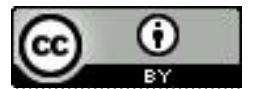




\title{
Differences between work methods and gender in computer mouse use
}

\author{
by Jens Wahlström, RPT, ${ }^{1}$ Joakim Svensson, RPT, ${ }^{1}$ Mats Hagberg, MD, ${ }^{1}$ Peter W Johnson, PhD ${ }^{1,2}$
}

\begin{abstract}
Wahlström J, Svensson J, Hagberg M, Johnson PW. Differences between work methods and gender in computer mouse use. Scand J Work Environ Health 2000;26(5):390-397.

Objectives The aim of this study was to investigate whether gender or different methods of operating a computer mouse have an effect on performance and musculoskeletal load in the use of a computer mouse.

Methods Thirty experienced computer mouse users, 15 men and 15 women, participated in the study. Electromyography (right first dorsal interossei, right extensor digitorum and right and left trapezius), a force-sensing mouse, and subjective ratings were used to register muscular load. An electrogoniometer was used to register the wrist movements. The subjects worked with 3 different methods, their own, a wrist-based method and an arm-based method. Gender comparisons were made when the subjects used their own method.

Results The women worked with greater extension and range of motion and tended to work with a greater ulnar deviation of the wrist. They also applied higher forces to the mouse when expressed as a percentage of a maximum voluntary contraction and had higher muscular activity in the right extensor digitorum. When using the arm-based method, the subjects worked with greater wrist extension, had higher muscular activity in the right and left trapezius muscles, and had the highest ratings of perceived exertion in the neck and shoulder. The wrist-based method resulted in higher forces being applied to the sides of the mouse and the highest ratings of perceived exertion in the wrist and hand-fingers.

Conclusions Gender differences were found for musculoskeletal load, and for most of the measured variables the women worked with higher loads than the men. The work method affected performance and musculoskeletal load. Finally, subjective measures appeared to have some utility in characterizing muscular load.
\end{abstract}

Key terms electrogoniometer, electromyography, forces, gender differences, input device, subjective ratings, video display terminal, video display unit.

There has been a rapid increase in the use of nonkeyboard computer input devices, and today the market is filled with such devices, the most common being the computer mouse. Several studies have suggested that an increased prevalence of upper-extremity musculoskeletal symptoms is associated with computer mouse use (14). According to data collected in 1997 in Sweden (5), $49 \%$ of working men and $45 \%$ of working women use a computer mouse in their profession. In a comparison of users of visual display units (VDU), mouse users were shown to have more extreme work postures in ulnar deviation of the wrist and outward rotation of the shoulder than keyboard operators did (6). Studying mouse use is of importance since more extreme work postures may increase a computer operator's risk for developing discomfort in the neck, shoulder, elbow, and wrist. Differences between methods of using a computer mouse have not been well documented; however, Karlqvist et al (7) observed gender differences and found that women work with more extreme shoulder postures when using the mouse. Studies have also shown that differences in mouse location and work with or without forearm support affect exposure and muscular load $(8,9)$.

It is hypothesized that there may be differences in musculoskeletal load between work methods and gender in computer mouse use. The aim of this study was to investigate whether different methods of operating a computer mouse or gender have an effect on performance and musculoskeletal load in computer mouse use.

\section{Subjects and methods}

\section{Subjects}

Thirty subjects, 15 men and 15 women, volunteered to participate in the study. Subjects from various

1 Department of Occupational Medicine, Sahlgrenska University Hospital, Göteborg, Sweden.

2 Ergonomics Laboratory, University of California San Francisco and Berkeley, Berkeley California, United States.

Reprint requests to: Jens Wahlström, Department of Occupational Medicine, Sahlgrenska University Hospital, St Sigfridsgatan 85, SE-412 66 Götborg, Sweden. [E-mail: jens.wahlstrom@ymk.gu.se] 
occupations were recruited from 2 different sections of the Sahlgrenska University Hospital, Göteborg, Sweden, and they participated in the experimental session during paid worktime. The mean age was 34 (range 18-52) years for the men and 39 (range 22-60) years for the women. The subjects were all experienced mouse users with a mean experience of 51 (range 6-144) months of mouse use at work or at home. Prior to the study, they were given written and verbal information on the experimental procedures and were interviewed to assure they were in good health and free of musculoskeletal disorders. They all used their right hand to operate the computer mouse.

\section{Experimental procedures}

An adjustable VDU workstation was set up, and the subjects adjusted the table and chair to fit their needs. A Macintosh computer with a 13-inch color display and a 101-key keyboard was used. Typically, the subjects adjusted the chair so that their legs were well supported with their feet resting flat on the floor; the table was adjusted so that the mouse and keyboard were approximately at elbow level and the monitor was at a fixed height above the work surface. The subjects were instructed by the same person to use the following 3 methods to operate the mouse: (i) a wrist-based method (WB) with which the forearm was fully supported on the desk and the mouse was moved by lifting and sweeping the mouse across the mouse-pad using the wrist, (ii) an arm-based method $(A B)$ with which only the wrist was supported on the work surface and the mouse was moved using movements initiated from the shoulder, and (iii) their own method (own). On the day before the study, in their own office, the subjects were instructed on how to perform the different methods and asked to practice and familiarize themselves with them. On the day of the measurement, they practiced at the experimental worksite to familiarize themselves with the equipment and to confirm that they performed the different work methods correct1y. The subjects performed a text editing task consisting of 8 paragraphs each containing 5 lines of 12-point Courier text. In each line, at a random location, 1 to 4 characters were highlighted using colored text. The subjects were instructed to select the colored characters with the mouse and then delete the characters by hitting the delete key on the keyboard with the mouse-using hand. They all used their own work method first, after which the sequence with the wrist-based and arm-based methods was introduced using a latin square design.

\section{Characterizing the subjects' own work method}

Three items from an ergonomic checklist were used to characterize the subject's own work method with the mouse. These items were (i) how the forearm or wrist was supported (near the elbow at the proximal part of the forearm or distally at the wrist or hand), (ii) whether or not the computer mouse was lifted from the surface, and (iii) the type of arm movements (whole arm or wrist and fingers). To characterize each subject's own work method, video recordings were made simultaneously from 2 different angles. Two of the researchers (JW, MH) independently characterized the subjects into 1 of 3 different groups (arm-based, wrist-based or a hybrid of the two) as they used their own method. If the result of the researchers differed, which occurred only 6 out of 30 times, a 3rd researcher (JS) analyzed the video recordings and made a final characterization. To be categorized as a user of the arm-based method, a subject could not rest the forearm on the work surface and had to use the whole arm to move the computer mouse. To be categorized as a user of the wrist-based method, a subject had to support the forearm on the work surface and use wrist movements to lift and move the computer mouse repeatedly.

\section{Forces applied to the mouse}

So that the forces applied to the sides and button of the mouse could be measured, a force-sensing Apple ADBII mouse was used. The force-sensing mouse was fully operational and similar in weight, feel, and appearance to a regular Apple ADBII mouse. The validity and measurement accuracy of the force-sensing mouse has been discussed elsewhere $(10,11)$. A portable computer instrumented with a PCMCIA data acquisition card (National Instruments, DAQCard 700, Austin, Texas, USA) was used to collect and store the force data. The force signals from the mouse were amplified using a portable amplifier (model 1210, FFA, Bromma, Sweden) and stored at $60 \mathrm{~Hz}$ on the hard disk of the computer. The force data were analyzed using a program written in Labview 4.0 (National Instruments, Austin, Texas, USA). The program identified each time the mouse was used, called a grip episode, and kept track of idle periods, which were defined as any period the mouse was not used for 1 second or longer. For each grip episode the program calculated the mean force, peak force, and grip duration.

\section{Maximum voluntary forces applied to the mouse}

The maximum forces the subjects could apply to the sides and button of the mouse were measured after the experiment. The subjects were asked to apply maximum voluntary contractions (MVC) to the sides and button of an Apple ADBII mouse instrumented with load cells (Pinchmeter, Greenleaf Medical, Palo Alto, California, USA). They were instructed to grip the mouse during the contractions the same way they gripped the mouse during the experiment. The contractions applied to the sides and button of the mouse were measured separately. Each subject applied 3 maximum voluntary contractions to the 
sides and the button of the mouse, and the highest force applied to each location was chosen as the subject's MVC. If the difference between the highest and second highest MVC was greater than $10 \%$, additional data were collected to verify the maximum.

\section{Wrist movements}

A 2-axis electrogoniometer (Model XM65, Penny \& Giles Biometrics, Blackwood, Wales) and a data logger (Model DL 1001, Penny \& Giles Biometrics, Blackwood, Wales) were used for recording flexion and extension and the radial and ulnar deviation angles of the right wrist. The sampling frequency was $20 \mathrm{~Hz}$. The reference (zero) position of the goniometer system was recorded when the subject sat at the workstation with the arm fully pronated in front and the hand flat in a neutral radial and ulnar position on the work surface (12). Goniometry data were analyzed using a program written in Labview 4.0. The program calculated the mean angles, mean velocities, mean power frequency, and the range of motion for both flexion-extension and radial-ulnar deviation. The mean power frequency was defined as the center of gravity for the power spectrum, and it has earlier been used as a measure for repetitiveness (13). The range of motion was defined as the difference between the 95 th and the 5 th percentile of the wrist angles (13).

\section{Muscular load}

Muscle activity from 4 separate muscles was recorded at $1 \mathrm{kHz}$ using a commercial electromyographic (EMG) system (model ME3000P, Mega Electronics Ltd, Kuopio, Finland). The muscles examined were the right first interossei, the right extensor digitorum, and the pars descendent of the right and left trapezius muscles. The electrodes for the first interossei and the extensor digitorum were placed as recommended by Perotto et al (14), and those for the trapezius were positioned as recommended by Mathiassen et al (15). Self-adhesive surface electrodes (M-00-S, Medicotest AS, Copenhagen, Denmark) were placed in pairs with a $35-\mathrm{mm}$ interelectrode distance. For the first interossei muscle, the electrodes were modified (cut) and resulted in an interelectrode distance of $25 \mathrm{~mm}$. Before the electrodes were attached, the skin was dry shaved and cleaned with alcohol. At the beginning of the recordings the subjects performed standardized maximum voluntary contractions to obtain the maximal voluntary electrical activity of the first interossei and the extensor digitorum muscles. Voluntary electrical activity in the first interossei and the extensor digitorum muscles were set with maximum static contraction against manual resistance for a minimum of 3 seconds. The reference voluntary electrical activity in the pars descendent of the right and left trapezius muscles was set with arms flexed 90 degrees, thumbs up, and a 1-kg dumbbell in each hand for a minimum of 3 seconds. A 3 -second sampling window was used to calculate the average electrical activity during the maximal and reference contractions. The raw data were recorded online using a portable computer and monitored in real-time for quality control. The EMG signal was smoothed using a root mean square (rms) procedure with an averaging moving window of $100 \mathrm{~ms}$. Data were analyzed using ME3000P software, version 1.5 (Mega Electronics Ltd, Kuopio, Finland), and the median EMG signal was calculated for each subject.

\section{Subjective ratings}

Before the experiment, the subjects rated perceived exertion using a modified Borg scale ranging from 0 to 14 (16) for the following 5 body regions; neck-shoulder (scapular), right shoulder (upper arm), right forearm, right wrist, and right hand-fingers. After the use of each work method, they rated their perceived exertion again. The ratings of perceived exertion (difference between perceived exertion after the 3 different conditions and before the experiment) were compared between work methods and between genders.

Besides the perceived exertion ratings, the subjects also rated their overall comfort with each work method on a scale graded from -4 (poor comfort) to +4 (excellent comfort).

\section{Productivity}

The total time and numbers of errors were used as a measure of productivity when the subjects used each of the different work methods. The total time was defined as the time it took the subject to complete the task from beginning to end. Errors were defined as any occurrence where the subject failed to highlight the text that was to be deleted. Errors were manually recorded by having one of the experimenters unobtrusively observe the subjects from behind and record each time an error occurred.

\section{Statistics}

Before the statistical tests, the data were tested for normality. They were analyzed using repeated-measures analyses of variance (JMP 3.2,SAS Institute Inc, Cary, North Carolina, USA). Descriptive data have been presented as means with the standard error of the mean (SEM). The comparisons between the work methods were performed using Tukey-adjusted t-tests (paired observations), and likewise-adjusted $95 \%$ confidence intervals $(95 \% \mathrm{CI})$ of the differences between the means were calculated. Gender comparisons were made using t-tests (2 independent groups) and were only performed on the data when subjects used their own work method. Due to technical problems, the results of 1 man were excluded from the analysis of wrist postures, and the results of another were excluded from the analysis of muscular load. 


\section{Results}

The gender comparisons are presented in table 1, and the method comparisons can be found in table 2 .

\section{Differences between genders}

The women applied almost twice the force of the men to the button of the mouse, when expressed as the percentage of the MVC (\%MVC). No gender differences were found when the forces were expressed as newtons. When operating the mouse, the women worked with greater extension and greater ulnar deviation and had a greater range of motion in the wrist when compared with the men. The women also had higher wrist velocities, but the men's wrist movements had a higher mean power frequency, the greatest differences occurring in flexion-extension. There were gender differences in muscular activity in the first interossei and extensor digitorum muscles, the women working with higher muscular activity than the men. The gender differences for the pars descendent of the left and right trapezius muscles were inconsistent, although there was a tendency for the men to have higher muscular activity (as percentage of the reference voluntary electrical activity) in the shoulder than the women. Small differences were found in the perceived exertion, although the women tended to rate it higher. The women were prone to have more errors and performed the task slightly faster, but these differences were negligible. When comfort was rated, no gender differences were found. The mean maximum force the men applied to the button and sides of the mouse was 60.4 (SEM 3.86) N and 98.6 (SEM 5.72) N, respectively. The mean maximum force of the women was 41.4 (SEM 1.73) $\mathrm{N}$ and 64.4 (SEM 2.54) $\mathrm{N}$, respectively.

\section{Differences between methods}

When using the wrist-based method, the subjects applied more force to the sides of the mouse than with the other methods, but the differences were smaller when the forces applied to the button of the mouse were compared. Differences between the methods were found for all the goniometric variables, the largest being for greater extension of the wrist and less range of motion, in radialulnar deviation, with the arm-based method and higher velocities with the wrist-based method. Muscular activity in the pars descendent of the right and left trapezius muscles was dependent on the work method, the highest occurring when the subjects worked with the arm-based method and the lowest being measured with the wristbased method. Only small differences in the first interossei and the extensor digitorum muscles were found between the work methods. Differences in perceived exertion were found between the work methods for all the body regions studied. After the wrist-based method, the perceived exertion was rated highest for the forearm, wrist, and hand-fingers, and after the arm-based method the highest ratings were given to the neck-shoulder, shoulder, and forearm. The subjects rated their own work method as the most comfortable, and the arm-based method as the least comfortable. With the wrist-based method the duration to complete the task was longer than with

Table 1. Gender differences in applied forces ( $N=15$ men and 15 women), wrist position data ( $N=14$ men and 15 women) and muscular activity ( $\mathrm{N}=14$ men and 15 women), including the mean differences and $95 \%$ confidence intervals $(95 \% \mathrm{Cl}$ ) of the differences. (SEM = standard error of the mean, $\mathrm{MVC}=$ maximal voluntary contraction, $\mathrm{ROM}=$ range of motion, $\mathrm{MPF}=$ mean power frequency, $\mathrm{FDI}=$ right first interossei muscle, MVE = maximal voluntary electrical activity, $E D=$ right extensor digitorum muscle, RTRAP = pars descendent of the right trapezius msucle, LTRAP = par descendent of the left trapezius muscle, RVE = reference voluntary electrical activity)

\begin{tabular}{|c|c|c|c|c|c|c|}
\hline \multirow[t]{2}{*}{ Variable } & \multicolumn{2}{|c|}{ Men } & \multicolumn{2}{|c|}{ Women } & \multicolumn{2}{|c|}{ Differences } \\
\hline & Mean & SEM & Mean & SEM & Mean & $95 \% \mathrm{Cl}$ \\
\hline Side mean force (\%MVC) & 0.82 & 0.08 & 0.97 & 0.10 & -0.15 & $-0.42-0.12$ \\
\hline Side peak force (\%MVC) & 1.44 & 0.15 & 1.74 & 0.21 & -0.30 & $-0.82-0.21$ \\
\hline Button mean force (\%MVC) & 1.20 & 0.09 & 1.85 & 0.16 & -0.65 & $-1.03--0.26$ \\
\hline Button peak force (\%MVC) & 2.92 & 0.30 & 4.62 & 0.45 & -1.69 & $-2.81--0.58$ \\
\hline Position (degrees), flexion-extension ${ }^{\mathrm{a}}$ & 25.9 & 1.6 & 30.3 & 1.5 & -4.4 & $-8.9-0.02$ \\
\hline ROM(degrees), flexion-extension & 16.8 & 1.0 & 21.7 & 1.6 & -4.9 & $-8.8--1.0$ \\
\hline Velocity (degrees/s), flexion-extension & 14.5 & 0.7 & 14.8 & 1.5 & -0.3 & $-3.8-3.1$ \\
\hline MPF $(\mathrm{Hz})$, flexion-extension & 0.80 & 0.04 & 0.57 & 0.04 & 0.23 & $0.11-0.35$ \\
\hline Position (degrees), deviation ${ }^{a}$ & 7.2 & 1.5 & 11.2 & 2.0 & -4.0 & $-9.2-1.1$ \\
\hline ROM(degrees), deviation & 15.9 & 1.7 & 21.8 & 2.1 & -5.9 & $-11.5--0.3$ \\
\hline Velocity (degrees/s), deviation & 8.5 & 0.8 & 11.2 & 1.1 & -2.6 & $-5.4-0.2$ \\
\hline MPF $(\mathrm{Hz})$, deviation & 0.43 & 0.03 & 0.43 & 0.03 & 0.005 & $-0.08-0.09$ \\
\hline FDI (\%MVE) & 7.6 & 1.7 & 11.3 & 2.2 & -3.6 & $-9.3-2.1$ \\
\hline ED (\%MVE) & 7.6 & 0.7 & 11.3 & 1.1 & -3.7 & $-6.4--0.9$ \\
\hline RTRAP (\%RVE) & 30.9 & 6.4 & 26.5 & 4.0 & 4.5 & $-10.9-19.8$ \\
\hline LTRAP (\%RVE) & 13.7 & 4.0 & 15.8 & 4.5 & -2.1 & $-14.5-10.3$ \\
\hline
\end{tabular}

a Positive values were used for extension and ulnar deviation. 
Table 2. Method differences in applied forces $(N=30)$, wrist position data $(N=29)$, muscular activity $(N=29)$, and ratings of perceived exertion (RPE) ( $\mathrm{N}=30)$ including the mean differences and the $95 \%$ confidence intervals $(95 \% \mathrm{Cl})$ of the differences. ( $\mathrm{SEM}=$ standard error of the mean, $\mathrm{ROM}=$ range of motion, $\mathrm{MPF}=$ mean power frequency, $\mathrm{FDI}=$ right first interossei muscle, $\mathrm{MVE}=$ maximal voluntary electrical activity, $\mathrm{ED}=$ right extensor digitorum muscle, RTRAP = pars descendent of the right trapezius muscle, LTRAP = par descendent of the left trapezius muscle, RVE = reference voluntary electrical activity)

\begin{tabular}{|c|c|c|c|c|c|c|c|c|c|c|}
\hline \multirow[t]{3}{*}{ Variable } & \multicolumn{6}{|c|}{ Method } & \multicolumn{4}{|c|}{ Comparison } \\
\hline & \multicolumn{2}{|c|}{ Own } & \multicolumn{2}{|c|}{$A B$} & \multicolumn{2}{|c|}{ WB } & \multirow[t]{2}{*}{ Methods } & \multicolumn{3}{|c|}{ Difference } \\
\hline & Mean & SEM & Mean & SEM & Mean & SEM & & Mean & $95 \% \mathrm{Cl}$ & \\
\hline \multirow[t]{3}{*}{ Side mean force $(\mathbb{N})$} & 0.69 & 0.05 & 0.63 & 0.05 & 0.97 & 0.06 & Own - wrist-based & -0.28 & $-0.40-$ & -0.16 \\
\hline & 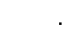 & 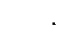 & 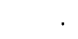 & & & & Own - arm-based & 0.06 & $-0.06-$ & 0.18 \\
\hline & & & & & & & Wrist-based - arm-based & 0.34 & $0.22-$ & 0.46 \\
\hline \multirow[t]{3}{*}{ Side peak force (N) } & 1.23 & 0.09 & 1.08 & 0.09 & 1.71 & 0.11 & Own - wrist-based & -0.48 & $-0.70-$ & -0.25 \\
\hline & & . & & & 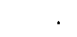 & & Own - arm-based & 0.15 & $-0.07-$ & 0.38 \\
\hline & & & & & & & Wrist-based - arm-based & 0.63 & $0.40-$ & 0.86 \\
\hline \multirow[t]{3}{*}{ Button mean force $(\mathrm{N})$} & 0.72 & 0.04 & 0.69 & 0.04 & 0.61 & 0.03 & Own - wrist-based & 0.11 & $0.05-$ & 0.17 \\
\hline & & & & & & & Own - arm-based & 0.04 & $-0.02-$ & 0.09 \\
\hline & & & & & & & Wrist-based - arm-based & -0.08 & $-0.13-$ & -0.02 \\
\hline Button peak force $(\mathrm{N})$ & 1.78 & 0.11 & 1.79 & 0.12 & 1.74 & 0.10 & Own - wrist-based & 0.04 & $-0.12-$ & 0.19 \\
\hline & & . & & & 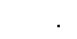 & & Own - arm-based & -0.01 & $-0.17-$ & 0.14 \\
\hline & & & & & & & Wrist-based - arm-based & -0.05 & $-0.20-$ & 0.11 \\
\hline Position (degrees), flexion-extension ${ }^{a}$ & 28.2 & 1.15 & 35.0 & 1.24 & 29.1 & 1.11 & Own - wrist-based & -0.9 & $-3.2-$ & 1.4 \\
\hline & & & & & & & Own - arm-based & -6.8 & $-9.1-$ & -4.5 \\
\hline & & & & & & & Wrist-based - arm-based & -5.9 & $-8.2-$ & -3.6 \\
\hline ROM (degrees), flexion-extension & 19.4 & 1.04 & 21.7 & 1.26 & 21.0 & 1.25 & Own - wrist-based & -1.6 & $-4.2-$ & 0.9 \\
\hline & & & . & & . & . & Own - arm-based & -2.3 & $-4.9-$ & 0.3 \\
\hline & & & & & 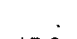 & & Wrist-based - arm-based & -0.6 & $-3.2-$ & 1.9 \\
\hline Velocity (degrees/s), flexion-extension & 14.7 & 0.83 & 13.9 & 0.76 & 15.8 & 0.85 & Own - wrist-based & -1.1 & $-2.4-$ & 0.2 \\
\hline & & & & . & & & Own-arm-based & 0.8 & $-0.5-$ & 2.1 \\
\hline & & & & & & & Wrist-based - arm-based & 1.9 & $0.6-$ & 3.2 \\
\hline MPF $(\mathrm{Hz})$, flexion-extension & 0.68 & 0.04 & 0.56 & 0.02 & 0.68 & 0.03 & Own - wrist-based & 0.00 & $-0.06-$ & 0.07 \\
\hline & . & . & . & 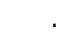 & 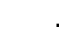 & & Own - arm-based & 0.12 & $0.05-$ & 0.18 \\
\hline & & & & & & & Wrist-based - arm-based & 0.11 & $0.05-$ & 0.18 \\
\hline Position (degrees), deviation ${ }^{\mathrm{a}}$ & 9.3 & 1.29 & 9.1 & 1.40 & 8.9 & 1.25 & Own - wrist-based & 0.4 & $-1.1-$ & 1.9 \\
\hline & . & " & & $\cdot$ & . & . & Own - arm-based & 0.1 & $-1.3-$ & 1.6 \\
\hline & & & & 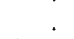 & 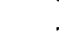 & & Wrist-based - arm-based & -0.3 & $-1.7-$ & 1.2 \\
\hline ROM (degrees), deviation & 19.0 & 1.46 & 15.7 & 1.05 & 21.2 & 1.54 & Own - wrist-based & -2.2 & $-5.1-$ & 0.7 \\
\hline & . & 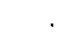 & 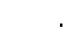 & . & & . & Own - arm-based & 3.3 & $0.4-$ & 6.2 \\
\hline & & & & & & & Wrist-based - arm-based & 5.5 & $2.6-$ & 8.4 \\
\hline Velocity (degrees/s), deviation & 9.9 & 0.72 & 8.5 & 0.44 & 11.1 & 0.77 & Own - wrist-based & -1.2 & $-2.5-$ & 0.2 \\
\hline & . & & . & . & . & . & Own-arm-based & 1.4 & $0.1-$ & 2.8 \\
\hline & & & & & & & Wrist-based - arm-based & 2.6 & $1.3-$ & 4.0 \\
\hline MPF (Hz), deviation & 0.43 & 0.02 & 0.45 & 0.02 & 0.45 & 0.02 & Own-wrist-based & -0.02 & $-0.07-$ & 0.04 \\
\hline & . & . & - & . & . & . & Own - arm-based & -0.02 & $-0.08-$ & 0.04 \\
\hline & & & & & & & Wrist-based - arm-based & -0.00 & $-0.06-$ & 0.05 \\
\hline FDI (\%MVE) & 9.5 & 1.41 & 10.0 & 1.64 & 9.5 & 1.53 & Own - wrist-based & 0 & $-1.10-$ & 1.10 \\
\hline & . & & & . & . & . & Own - arm-based & -0.45 & $-1.55-$ & 0.65 \\
\hline & & & & & & & Wrist-based - arm-based & -0.45 & $-1.55-$ & 0.65 \\
\hline ED (\%MVE) & 9.5 & 0.74 & 10.0 & 0.76 & 9.9 & 0.68 & Own - wrist-based & -0.38 & $-1.25-$ & 0.49 \\
\hline & . & . & & 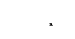 & . & & Own - arm-based & -0.55 & $-1.42-$ & 0.32 \\
\hline & & & & & & & Wrist-based - arm-based & -0.17 & $-1.04-$ & 0.70 \\
\hline RTRAP (\%RVE) & 28.6 & 3.69 & 52.4 & 5.57 & 21.4 & 2.77 & Own - wrist-based & 7.2 & $-2.4-$ & 16.7 \\
\hline & . & . & . & & . & . & Own - arm-based & -23.8 & $-33.3-$ & -14.2 \\
\hline & & & & & & & Wrist-based - arm-based & -30.9 & $-40.5--$ & -21.4 \\
\hline LTRAP (\%RVE) & 14.8 & 2.97 & 22.1 & 3.55 & 9.3 & 3.11 & Own - wrist-based & 5.5 & $-1.2-$ & 12.2 \\
\hline & & & 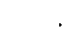 & & 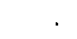 & . & Own - arm-based & -7.3 & $-14.0-$ & -0.6 \\
\hline & & & & & & & Wrist-based - arm-based & -12.8 & $-19.5-$ & -6.1 \\
\hline Neck-shoulder (RPE, scale step) & 0.3 & 0.4 & 2.0 & 0.6 & 0.4 & 0.5 & Own - wrist-based & -0.1 & $-1.2-$ & 1.1 \\
\hline & . & . & . & & . & & Own - arm-based & -1.7 & $-2.8-$ & -0.5 \\
\hline & & & & & & & Wrist-based - arm-based & -1.6 & $-2.7-$ & -0.5 \\
\hline Shoulder (RPE, scale step) & 0.6 & 0.3 & 3.8 & 0.5 & 1.4 & 0.5 & Own - wrist-based & -0.9 & $-1.8-$ & 0.1 \\
\hline & & & . & & & & Own - arm-based & -3.2 & $-4.2-$ & -2.3 \\
\hline & & & & & & & Wrist-based - arm-based & -2.4 & $-3.3-$ & -1.4 \\
\hline Forearm (RPE, scale step) & 1.1 & 0.3 & 2.9 & 0.6 & 2.6 & 0.5 & Own - wrist-based & -1.5 & $-2.6-$ & -0.4 \\
\hline & . & & . & . & . & & Own - arm-based & -1.8 & $-2.9-$ & -0.6 \\
\hline & & & & & & & Wrist-based - arm-based & -0.3 & $-1.4-$ & 0.9 \\
\hline Wrist (RPE, scale step) & 0.9 & 0.3 & 1.6 & 0.3 & 3.1 & 0.5 & Own - wrist-based & -2.2 & $-3.2-$ & -1.2 \\
\hline & . & & . & 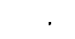 & & & Own - arm-based & -0.6 & $-1.7-$ & 0.4 \\
\hline & & & & & & & Wrist-based - arm-based & 1.6 & $0.5-$ & 2.6 \\
\hline Hand-fingers (RPE, scale step) & 0.9 & 0.2 & 1.5 & 0.3 & 2.1 & 0.4 & Own - wrist-based & -1.2 & $-2.1-$ & -0.3 \\
\hline & . & . & & . & . & & Own - arm-based & -0.5 & $-1.4-$ & 0.3 \\
\hline & . & . & & . & . & & Wrist-based - arm-based & 0.7 & $-0.2-$ & 1.5 \\
\hline
\end{tabular}

a Positive values were used for extension and ulnar deviation. 
the other 2 methods. According to the video observations used to characterize each subject's own work method, 9 subjects used an arm-based method, 7 used a wrist-based method, and 14 used a hybrid method (primarily a wristbased method where the mouse was not lifted off the mouse pad).

\section{Discussion}

\section{Differences between genders}

The main finding of this study was the gender differences in work with the computer mouse. Women worked with greater wrist extension, greater ulnar deviation, and a greater range of motion and had higher wrist velocities. The smaller stature of the women and the fixed size of the devices used may explain part of this difference. The fixed size of the keyboard may have caused more outward rotation of the shoulder and ulnar deviation of the wrist, and the fixed height of the mouse could have resulted in more wrist extension among the women than among the men. The only variable for which the men's wrist movements exceeded the women's was the mean power frequency in flextion-extension. This difference could be explained by the smaller range of motion that the men worked with in that it resulted in a greater frequency of repetitive movements about the wrist. Women also operated the mouse with higher muscular activity in the extensor digitorum muscle and tended to have higher muscle activity in the first interossei muscle. They also applied higher forces to the computer mouse when expressed as the percentage of the MVC. Similar results for muscular activity $(7,8)$ and forces $(17,18)$ have been reported by other researchers. These finding may be related to the lower muscular strength of women and anthropometric differences, which influence biomechanical loads. Fixed button actuation forces in combination with strength differences is a possible explanation for why women applied more relative (\%MVC) force than the men. Another reason could be that women have smaller hands, which result in higher relative exertion needed to grip the mouse.

In a review of epidemiologic findings, Punett \& Bergqvist (19) stated that a woman "appears to be consistently associated with higher occurrences of several upper extremity and neck muscle discomforts among VDU users" [p 120]. No definite explanations were found in the reviewed studies, but constitutional and work situation differences were mentioned as possibilities. Tittiranonda and her co-workers (20) suggested that "small anthropometric dimensions (i.e., hand and height) may cause women to work in postures or at higher relative muscle forces, which cause greater mechanical stresses, than men" [p 33]. Finally, it is worth noting that the gender differences observed were consistent across all 3 methods of operating the mouse.

\section{Differences between methods}

Two basic elements characterize work technique, the method or systems of methods used to carry out a task (21) and the individual motor performance of the task (22). The "method" in our study was represented by the 3 different ways of operating the mouse. "Individual performance" focuses on the individual variations in how a given "method" is executed. There were interindividual differences in all the measured variables, and this variation can be explained by differences in anthropometry, strength, and motivational factors (23).

The trapezius muscle was the most sensitive muscle for detecting differences in muscular activity between methods. When looking for the extremes in the variables measured, the arm-based method was distinguished from the other 2 methods, as the subjects worked with higher muscular activity in the right trapezius muscle. The other muscles showed little differences in the comparison of methods, at least at the group level of muscular activity. Previous studies have shown that operating the computer mouse without support of the forearm increases the load on the trapezius muscle $(24,25)$.

The velocity of the wrist (flexion-extension and radial-ulnar deviation) was higher when the subjects used the wrist-based method. Only small differences were found in mean power frequency between the methods, and it varied from 0.43 to $0.45 \mathrm{~Hz}$ in radial-ulnar deviation and from 0.56 to $0.68 \mathrm{~Hz}$ in flexion-extension. In a study by Karlqvist et al (25), with computer mouse operators, the mean power frequency was between 0.23 and $0.28 \mathrm{~Hz}$ in flexion-extension and between 0.21 and 0.24 $\mathrm{Hz}$ in radial-ulnar deviation. The specific task in the present study resulted in higher mean power frequencies (ie, higher repetitiveness) when compared with the corresponding values of the Karlqvist et al study (25). This difference may have been due to fewer pauses or the repetitive nature of the task.

The forces applied to the sides of the mouse formed the most sensitive force variable for detecting differences between the methods. In a study by Johnson et al (17), whose subjects performed a similar text editing task, the forces applied to the sides of the mouse were found to be highly correlated with those of regular work $(\mathrm{r}=0.89)$. Whether these low forces are risk factors for developing symptoms is unknown, but the combination of high repetitiveness and long duration could be a plausible factor in the development of musculoskeletal symptoms.

Changes in subjective ratings, after a short duration of physical exposure, appear to be of some value for detecting differences in perceived exertion between the different methods of operating the mouse. When the 3 methods were compared, the greatest increases in perceived 
exertion occurred in the neck-shoulder and shoulder regions after the use of the arm-based method. After the wrist-based method the greatest increases in perceived exertion were reported for the wrist and hand-fingers. The subjective ratings of perceived exertion often mirrored the trends observed in the objective physical findings, and similar trends have previously been reported (7).

The differences between the methods could, in part, be due either to a difference between the number of men and women who use each method as their "own" method or to the distribution of arm-based and wrist-based users; however, the distribution in both cases was relatively balanced so the differences were probably attributable to differences between the methods themselves.

\section{Limitations of the study}

The subjects were instructed to use work methods that they did not use in their regular work. We tried to limit the effect of unfamiliar motion patterns with training sessions before the test. The data collection for each method was made during approximately $3-4$ minutes, which may be a short time, but all the subjects were experienced computer mouse operators and should have had few, if any, initial difficulties in performing the task. The task performed was text editing, and the generalizability to other worktasks is limited. However, it has been shown that the forces applied to the computer mouse during text editing tasks are highly correlated with regular worktask forces (17). Finally, only 1 type of mouse was tested in this study, and it would be of interest to determine whether the same trends occur with other mice.

\section{Concluding remarks}

Gender differences were found for musculoskeletal load, and, for most of the measured variables, the women worked with higher loads than the men. The work method affected performance and musculoskeletal load. Finally, subjective measures appear to have some utility in characterizing muscular load.

\section{References}

1. Fogleman M, Brogmus G. Computer mouse use and cumulative trauma disorders of the upper extremities. Ergonomics 1995;38(12):2465-75.

2. Franzblau A, Flaschner D, Albers JW, Blitz S, Werner R, Armstrong T. Medical screening of office workers for upper extremity cumulative trauma disorders. Arch Environ Health 1993;48(3):164-70.

3. Jensen C, Borg V, Finsen L, Hansen K, Juul-Kristensen B, Christensen $\mathrm{H}$. Job demands, muscle activity and musculoskeletal symptoms in relation to work with the computer mouse. Scand J Work Environ Health 1998;24(5, special section): $418-24$.
4. Karlqvist L, Hagberg M, Köster M, Wenemark M, Ånell R Musculoskeletal symptoms among computer-assisted design (CAD) operators and evaluation of a self assessment questionnaire. Int J Occup Environ Health 1996;2:185—94.

5. Statistics Sweden. Arbetsmiljön 1997 [The work environment 1997]. Stockholm: Statistics Sweden, 1997. Report no AM 68 SM 9801.

6. Karlqvist L, Hagberg M, Selin K. Variation in upper limb posture and movement during word processing with and without mouse use. Ergonomics 1994;37(7):1261 -7.

7. Karlqvist L, Bernmark E, Ekenvall L, Hagberg M, Isaksson A, Rostö T. Computer mouse and track-ball operation similarities and differences in posture, muscular load and perceived exertion. Int J Ind Ergon 1999;23:157—69.

8. Karlqvist LK, Bernmark E, Ekenvall L, Hagberg M, Isaksson A, Rostö T. Computer mouse position as a determinant of posture, muscular load and perceived exertion. Scand J Work Environ Health 1998;24(1):62-73.

9. Aarås A, Ro O. Workload when using a mouse as an input device. Int J Hum Comput Interaction 1997;9(2):105-18.

10. Johnson PW, Dropkin J, Hewes J, Rempel D. Office ergonomics: motion analysis of computer mouse usage. In: American Industrial Hygiene Association. Proceedings of the American Industrial Hygiene Conference and Exposition. Fairfax (VA): American Industrial Hygiene Association, 1993;12-3.

11. Johnson PW, Tal R, Smutz WP, Rempel DM. Fingertip forces measured during computer mouse operation: a comparison of pointing and dragging. In: Human Factors Association. Proceedings of the 12th Triennal Congress of the International Ergonomics Association; vol 2. Mississauga (ONT): Human Factors Association, 1994;208-10.

12. Greene WB, Heckman JD, editors. The clinical measurement of joint motion. Rosemont (IL): American Academy of Orthopaedic Surgeons, 1994.

13. Hansson G-Å, Balogh I, Ohlsson K, Rylander L, Skerfving S. Goniometer measurement and computer analysis of wrist angles and movements applied to occupational repetitive work. J Electromyographical Kinesiology 1996;6:23-35.

14. Perotto A. Anatomical guide for the electromyographer: the limbs and trunk. 3rd rev ed. Springfield (IL): Charles C Thomas, 1994.

15. Mathiassen SE, Winkel J, Hägg G. Normalization of surface EMG amplitude from the upper trapezius muscle in ergonomic studies - a review. J Electromyographical Kinesiology 1995;5:197-226.

16. Borg G. Psychophysical scaling with applications in physical work and the perception of exertion. Scand J Work Environ Health $1990 ; 16$ suppl 1:55-8.

17. Johnson PW, Hagberg M, Wigaeus Hjelm E, Rempel D. Exposure assessment: measuring and characterizing the forces applied to the computer mouse. In: Finnish Institute of Occupational Health. PREMUS-ISEOH' '98 3rd International Scientific Conference on Prevention of Work-Related Musculoskeletal Disorders; 13th International Symposium on Epidemiology in Occupational Health, 21-25 September 1998 Helsinki: Finnish Institute of Occupational Health, 1998;120.

18. Martin BJ, Armstrong TJ, Foulke JA, Natarajan S, Klinenberg E, Serina E, et al. Keyboard reaction force and finger flexor electromyograms during computer keyboard work. Hum Factors 1996;38(9):654-64.

19. Punnett L, Bergqvist U. Visual display unit work and upper extremity musculoskeletal disorders. Solna: National Institute for Working Life, 1997. Arbete och Hälsa, no 16.

20. Tittiranonda P, Burastero S, Rempel D. Risk factors for musc- 
uloskeletal disorders among computer users. Philadelphia (PA): Hanley \& Belufs Inc, 1999:17-38. Occupational Medicine: State of the Art Reviews, vol 14.

21. Hagner I-M, Hagberg M. Evaluation of two floor mopping work methods by measurement of load. Ergonomics 1989;32(4):401-8.

22. Kilbom $\AA$, Persson J. Work technique and its consequences for musculoskeletal disorders. Ergonomics 1987;30(2):2739.

23. Kjellberg K, Lindbeck L, Hagberg M. Method and performance: two elements of work technique. Ergonomics 1998; 41(6):798-816.
24. Aarås A, Fostervold KI, Ro O, Thoresen M, Larsen S. Postural load during VDU work: a comparison between various work postures. Ergonomics 1997;40(11):1255-68.

25. Karlqvist L, Hagberg M, Hansson G-Å, Huldt Mattsson G, Neelakantan, Skantze C, et al. God arbetsmiljö vid bildskärmsarbete med datormus i den grafiska branschen [Good working environments for graphic industry VDU operators using a mouse]. Solna: National Institute for Working Life, 1995. Arbete och Hälsa, no 21.

Received for publication: 30 June 1999 